\title{
Radiation damage monitoring of the ATLAS pixel detector
}

\author{
Sally Seidel ${ }^{* \dagger}$ \\ University of New Mexico \\ E-mail: seidelephys.unm.edu
}

\begin{abstract}
A measurement has been made of the radiation damage incurred by the ATLAS Pixel Detector barrel silicon modules from the beginning of operations through the end of 2012. This translates to hadronic fluence received over the full period of operation at energies up to and including 8 $\mathrm{TeV}$. The measurement is based on a per-module record of the silicon sensor leakage current. The results are presented as a function of integrated luminosity and compared to predictions by the Hamburg Model. This information can be used to predict limits on the lifetime of the Pixel Detector due to current, for various operating scenarios at the LHC.
\end{abstract}

24th International Workshop on Vertex Detectors

1-5 June 2015

Santa Fe, New Mexico, USA

\footnotetext{
* Speaker.

${ }^{\dagger}$ Representing the ATLAS Collaboration.
} 


\section{Introduction}

The ATLAS Pixel Detector [1] is constructed from planar silicon pixel modules arranged in a barrel and disk geometry. It is centered on the interaction point at the heart of the ATLAS Detector [2] at the Large Hadron Collider (LHC). A linear relationship between leakage current and hadronic particle fluence is expected to apply to the silicon pixel sensors and is given by $\Delta I=\alpha \cdot \Phi_{e q} \cdot V$, where $\Delta I$ is the difference in leakage current at fluence $\Phi_{e q}$ relative to the value before irradiation of the physical volume $V$, and $\alpha$ is the current-related damage coefficient [3]. The goal of this study was to measure the leakage currents of a representative sample of sensors in the ATLAS Pixel Detector in order to monitor and understand the sensor damage resulting from increasing radiation dose. The measured results are compared to predictions based on the Hamburg Model [4] in which reverse bias current is generated by the introduction of defect levels in the band gap due to non-ionizing energy loss (NIEL) of through-going particles. The defects migrate and recombine through several processes that result in both short-term beneficial annealing (which reduces the current) and long-term anti-annealing (which increases the current even in the absence of further radiation). The model can be calibrated to the data to predict the lifetime of the sensors and the Pixel Detector as a whole for various operating scenarios. Prior to 2015, the detector was instrumented as three barrels, specifically the Layer- 0 at $r=50.5 \mathrm{~mm}$, the Layer- 1 at $r=88.5$ $\mathrm{mm}$, and the Layer-2 at $r=122.5 \mathrm{~mm}$, comprising in total 1456 modules. The endcap areas are instrumented with three disks located at $z= \pm 495 \mathrm{~mm}, \pm 580 \mathrm{~mm}$ and $\pm 650 \mathrm{~mm}$ in each of the forward and backward regions, comprising an additional 288 modules. The Pixel Detector and the other elements of the ATLAS Inner Detector span a pseudorapidity range $|\eta|<2.5 .^{1}$ The pixel sensor modules are mounted on mechanical/cooling supports, called staves, in the barrel region. Thirteen modules are mounted on a stave with identical layouts for all layers. The temperature is maintained stable by an evaporative cooling system. The entire $1.7 \mathrm{~m}^{2}$ sensitive area of the Run 1 ATLAS pixel detector is covered with 1744 identical modules. Each module has an active surface of $6.08 \times 1.64 \mathrm{~cm}^{2}$. The details of the pixel sensor geometry and layout can be found in [1]. The radiation field in the region of the detector has been predicted [5] with the aid of the FLUKA [6] software package. The FLUKA transport code to cascade the particles through the ATLAS detector material was used together with PYTHIA 6.2 [7] or PHOJET [8], generating proton-proton minimum-bias events as an input to FLUKA [1]. We assume that the dominant radiation damage type is displacement defects in the bulk of the pixel sensor, initiated by hadronic species and caused by NIEL. Surface ionization is neglected in this treatment. Charged pions are expected to dominate the bulk damage for the radii covered by the Pixel Detector. Albedo neutrons originating in the outer ATLAS detectors also contribute. The resulting displacement defects increase the reverse leakage current, degrade the charge collection efficiency, and change the effective doping concentration which directly determines the depletion voltage. We define the effective fluence $\Phi_{e q}$ as the number of particles causing damage equivalent to that of $1 \mathrm{MeV}$ neutrons traversing $1 \mathrm{~cm}^{2}$ of a sensor's surface. The fluence $\Phi_{e q}$ accumulated by the ATLAS pixel

\footnotetext{
${ }^{1}$ ATLAS uses a right-handed coordinate system with its origin at the nominal interaction point (IP) in the center of the detector and the z-axis along the beam pipe. The x-axis points from the IP to the center of the LHC ring, and the $\mathrm{y}$-axis points upward. Cylindrical coordinates $(r, \phi)$ are used in the transverse plane, $\phi$ being the azimuthal angle around the beam pipe. The pseudorapidity is defined in terms of the polar angle $\theta$ as $\eta=\ln \tan (\theta / 2)$.
} 
detector, and measured in units of $\mathrm{cm}^{-2}$, is expected to be proportional to the integrated luminosity $\int \mathscr{L} d t$, measured in $\mathrm{pb}^{-1}$.

\section{Measured quantities}

The parameter $\alpha$ has been measured under a variety of conditions, for example [3]. The linear form applies to the leakage currents drawn by sensors past their beneficial annealing periods. At the beginning of data-taking and during beneficial annealing periods, the sensors draw currents at levels comparable to dark currents; thus any hardware implementations for leakage current measurement should provide access to the lowest current range possible. The reverse bulk generation current depends on the sensor temperature $T$. The current measurements $I(T)$ are normalized to the reference temperature $T_{R}\left(0^{\circ} C\right)$ with $I(T)=I\left(T_{R}\right) / R(T)$, where $\left.R(T)=\left(T_{R} / T\right)^{2} \cdot \exp \left[\frac{E_{g}}{2 k_{B}}\left(1 / T_{R}-1 / T\right)\right)\right]$, where $E_{g}=1.21 \mathrm{eV}$ is the energy of the silicon band gap, $k_{B}$ is the Boltzmann constant, and $T$ and $T_{R}$ are measured in $\mathrm{K}$ [9]. The mean pixel module temperature for all barrel pixel modules is shown versus time in Figure 1 [10]. There were a few exceptions when the temperature rose to ambient due to cooling interruptions and calibration scans. Temperature is read out from every module continuously and averaged over 30-minute intervals. Temperature data (as well as other slow control data) are committed into the ATLAS Detector Control System (DCS) database.

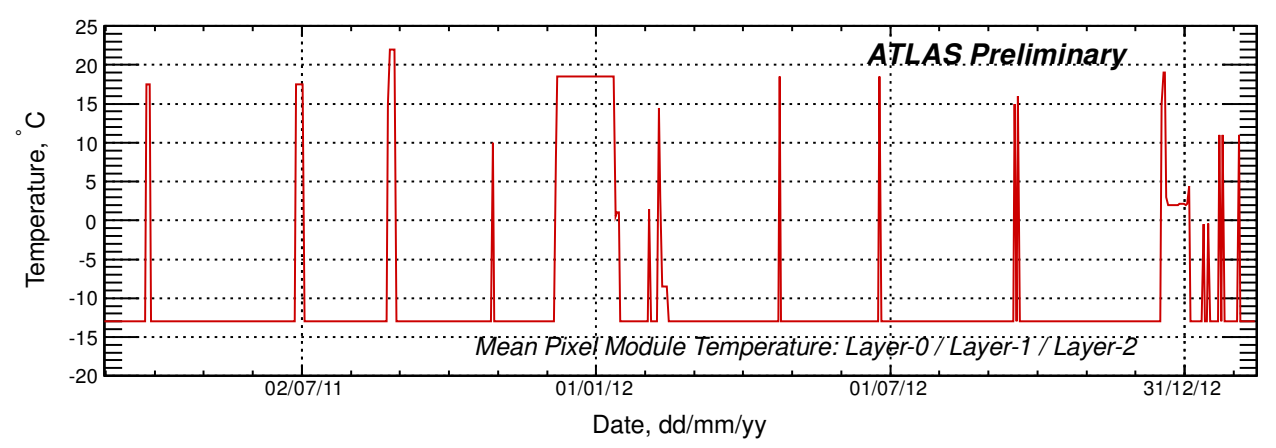

Figure 1: The mean temperature $\left({ }^{\circ} \mathrm{C}\right)$ of all barrel pixel modules, versus time (days).

\section{The ATLAS current measurement subsystem}

The ATLAS High Voltage Patch Panel 4 (HVPP4) serves as a fan-out point for the bias voltages delivered by Type II boards from Iseg [11] high voltage power supplies to the pixel modules. The leakage current is monitored at the pixel module granularity level by a Current Measurement Board (CMB) system. A CMB is mounted on every Type II fan-out board. The bias voltage to the sensors is provided by the Iseg channels. In the present data-taking era, when the radiation damage of the sensors is relatively low, 6 or 7 pixel modules in Layers 0,1 , and 2 are fed by one Iseg power supply channel. At some point after inversion of the sensors, before the current drawn by 6 or 7 pixel modules reaches the Iseg limit, power supplies can be added until the system provides one Iseg power supply channel per pair of pixel modules. The CMB system allows simultaneous 
measurement of the leakage currents in 4 pixel modules in the current range from $0.04 \mathrm{~mA}$ to $2 \mathrm{~mA}$ with a precision of better than $20 \%$ per module. ATLAS uses 21 CMBs in Layer-0, 16 in Layer1 , and 16 in Layer-2. The analog current measurements are digitized by the 64-channel ATLAS Embedded Local Monitoring board (ELMB) and sent via the Controller Area Network (CAN) bus to the DCS database (see [1] for details on the Type II fan-out board, ELMB, CAN, and DCS). The building block of the CMB circuit is a current to frequency converter optically coupled to a frequency to voltage converter as shown in Figure 2. Each CMB holds $4 \times 2$ current measurement circuits and provides the current measurement for 4 pixel modules. Two similar circuits with different gains are allocated per CMB channel serving the same module. The channels are isolated from each other and from the pixel module readout system. For each pixel module, the leakage current range $\left[10^{-8}-10^{-5}\right] \mathrm{A}$ is covered by the high-gain channel, and the range $\left[10^{-6}-2 \cdot 10^{-3}\right] \mathrm{A}$ is covered by the low-gain channel. The output $\mathrm{CMB}$ voltage range is defined by one of five available ELMB input voltage ranges, which are [0 - 25] mV, [0 - 100] mV, [0 - 1] V, [0 - 2.5] V, and [0 - 5] V. The output range [0 - 5] V is necessary to comply with the specifications of the digital ELMB board. The 16-bit ADC of the ELMB provides a resolution of (ELMB range)/(216 -1$)$. The range [0 - 1] $\mathrm{V}$ was used for data taken through 2011; the range was changed to [0 - 5] V in 2012 as the high-gain channels reached saturation, corresponding to pixel leakage currents greater than $10^{-5} \mathrm{~A}$. After the LHC shutdown of 2013-2014, the data-taking has resumed with the lowgain channels. Before installation, each CMB is calibrated offline assuming a linear model. The calibrated gains of the channels are stored in the database. The pedestals are re-calibrated in situ.

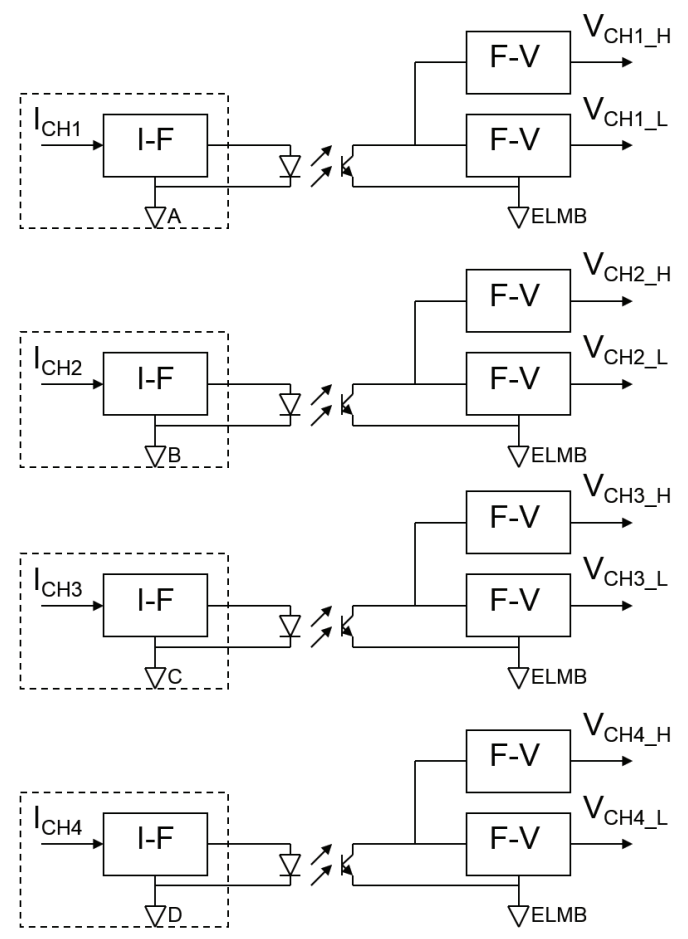

Figure 2: The Current Measurement Board circuit. 


\section{Current measurements, 2011-2012}

Figure 3 shows the leakage current recorded by the CMB system from the beginning of ATLAS data-taking through the end of 2012, as a function of integrated luminosity, for modules in all three Pixel Detector barrel layers [10]. (The post-2014 Insertable B-Layer has a separate system not described here.) Installation of the CMB system proceeded concurrently with early data-taking, and this is the reason that some Layer-2 data are missing from the early part of the run. The disks were instrumented with CMBs prior to the start of data-taking in 2015 (Run 2). The points at which the LHC and cooling were off, hence beneficial annealing of the silicon was unsuppressed, are apparent as discontinuities in this figure. This effect, producing the sawtooth features, is due to the increase of the effective doping concentration of the silicon due to annealing of acceptors [3, 4]. Figure 3 also includes a prediction [4] of the leakage current for the detailed ATLAS geometry and LHC luminosity profile based on the Hamburg Model and a prediction of the fluence $\Phi_{e q}$ by the FLUKA transport code. The predictions are uniform with respect to the azimuthal angle $\phi$ and assume a forward-backward symmetry with respect to the polar angle $\theta$, i.e. $\Phi_{e q}(\eta)=\Phi_{e q}(-\eta)$. At the end of Run 1 with $\int \mathscr{L} d t=30 \mathrm{fb}^{-1}$, the Layer-0 modules had received a total fluence $\Phi_{e q}=7.0 \cdot 10^{13} \mathrm{~cm}^{-2}$, while Layer-1 and Layer-2 had been irradiated to $\Phi_{e q}=3.0 \cdot 10^{13} \mathrm{~cm}^{-2}$ and $\Phi_{e q}=1.8 \cdot 10^{13} \mathrm{~cm}^{-2}$ respectively. The uncertainty of the leakage current predictions comprises the uncertainty of the calculations of the hadronic particle spectra initiated by pp interactions in the ATLAS detector and the uncertainty of the Hamburg model in describing radiation damage effects inflicted by the NIEL of hadrons crossing the volume of the silicon pixel sensors. Figure 4 shows the distribution in azimuthal angle of modules whose current is sampled by CMBs in the ATLAS Pixel Detector. Figure 5 shows the data for each of four quadrants in the azimuthal angle as they are defined by Figure 4 [10]. No systematic difference in module leakage current is observed with respect to the quadrant where the module is located. Figure 6 shows the instrumented module distribution, segmented into five sectors in pseudorapidity. The pseudorapidity acceptance of each sector of each layer is indicated. Figure 7 shows the leakage current data for each of the 5 ranges in pseudorapidity [10]. Figure 8 shows the current data versus pseudorapidity for the sectors defined by Figure 6, for integrated luminosity $25 \mathrm{fb}^{-1}$ [10]. The possible slight asymmetry of the data points in Figure 8 is thought to be due to the asymmetric beam profile. Yet the distribution is in agreement within the uncertainties with the leakage current predictions which are symmetrical according to the fluence simulation [5].

\section{Precision and systematic uncertainties}

The contributions to the uncertainty on the current include

- the CMB and ELMB precision on current measurements: $12 \%$ for both ranges.

- the number of current measurements (two per hour).

- the uncertainty on the luminosity, $1.8 \%$ in 2011 and $2.8 \%$ in 2012 [12].

- the temperature uncertainty, which, at less than $0.3^{\circ} \mathrm{C}$, contributes to the uncertainty on the current with $3.4 \%$. 


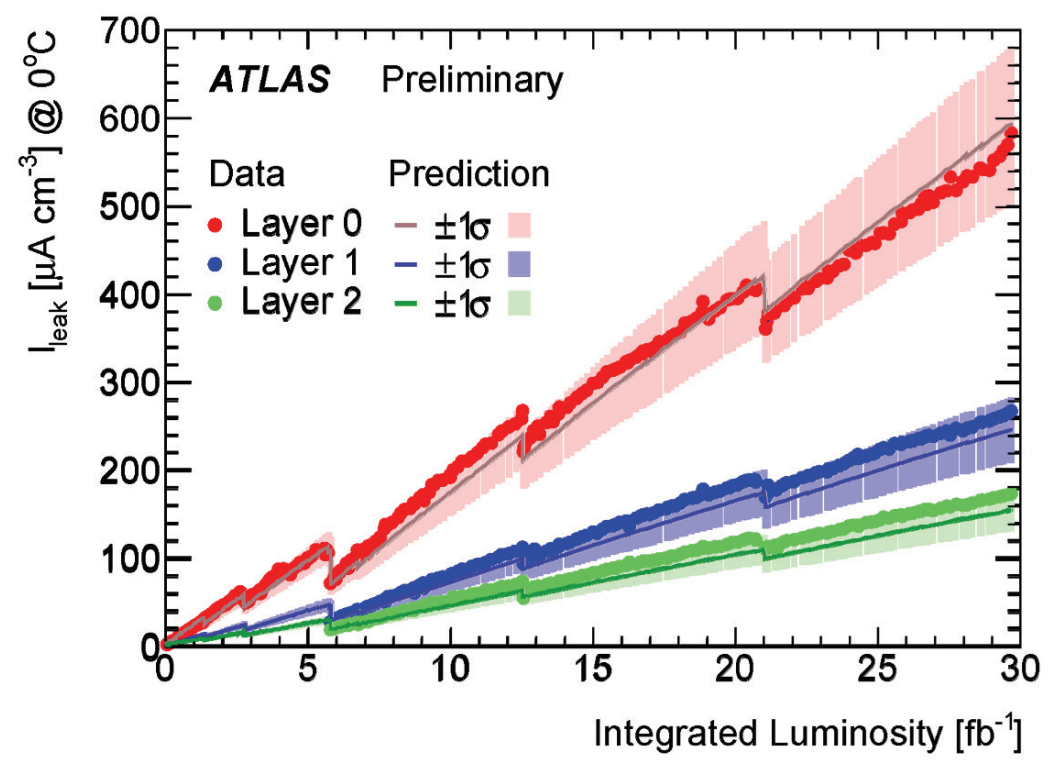

Figure 3: ATLAS pixel module leakage current versus integrated LHC luminosity. The currents are averaged over their layer for all modules equipped with Current Measurement Boards within the layer. The current is continuously monitored by the ATLAS Detector Control System. A prediction based on the Hamburg Model is included. Discontinuities are due to annealing during LHC and cooling stops.

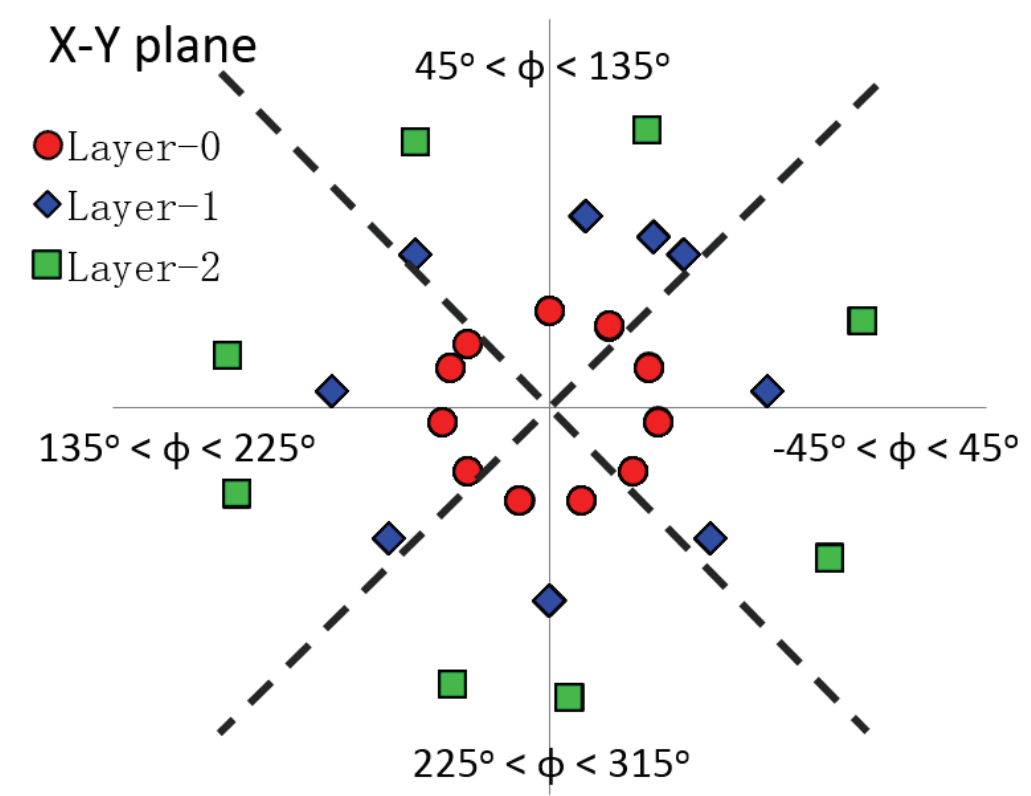

Figure 4: A diagram of the distribution of instrumented modules in each of four sectors of azimuthal angle. 

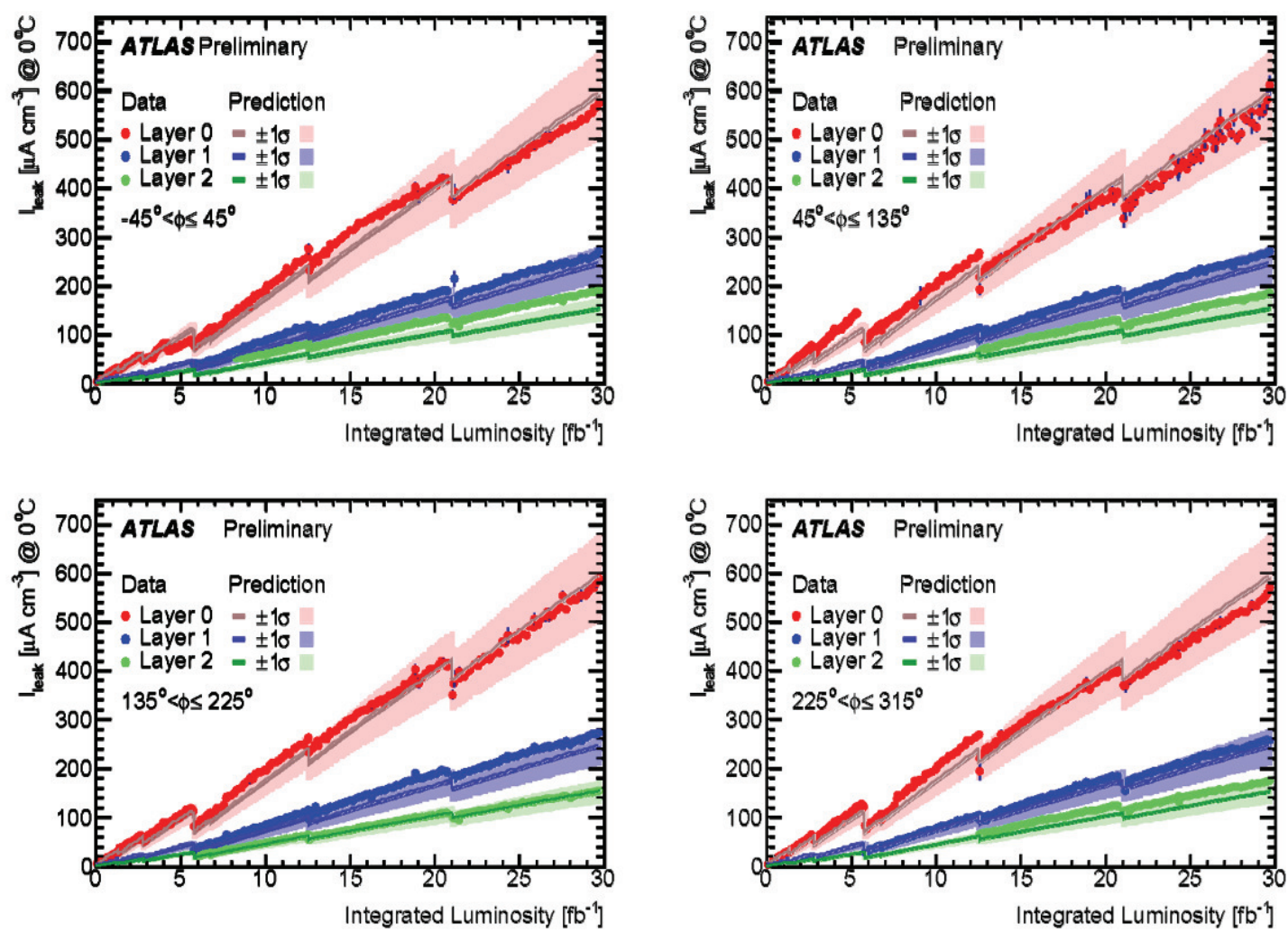

Figure 5: ATLAS pixel module leakage current in each of 4 quadrants in azimuthal angle, versus integrated LHC luminosity. The currents are averaged over their layer for all modules equipped with Current Measurement Boards within the layer. The current is continuously monitored by the ATLAS Detector Control System. Predictions based on the Hamburg Model are included. Discontinuities are due to annealing during LHC and cooling stops.

\section{Lifetime estimate}

The temperature-corrected current readings per module can be extrapolated to predict the amount of current the pixel modules will draw after a certain integrated luminosity has been collected with the ATLAS pixel detector. As leakage current can lead to excessive power and thermal runaway, limits on the module lifetime due to current can be determined from the limit on the bias voltage that can be applied. A single Iseg power supply channel can sustain a maximum current of $<4000 \mathrm{~mA}$ for two modules. There are other consequences of radiation-induced leakage current upon module lifetime, including the noise, bias, and threshold limitations of the front end chips as well as the finite capacity of the cooling system; while important, these are not treated here. We extrapolate the present rate of current increase with integrated luminosity, assuming that the modules will be exposed for 10 days to $20^{\circ} \mathrm{C}$ and otherwise maintained at $-13^{\circ} \mathrm{C}$ in future years. The model assumes that the bias voltage is raised throughout LHC operation as needed to keep the bulk fully depleted, until a maximum allowed voltage of $600 \mathrm{~V}$ is reached. Only the effects of proton runs are considered. Figure 9 shows the implied leakage current per module versus date and versus 


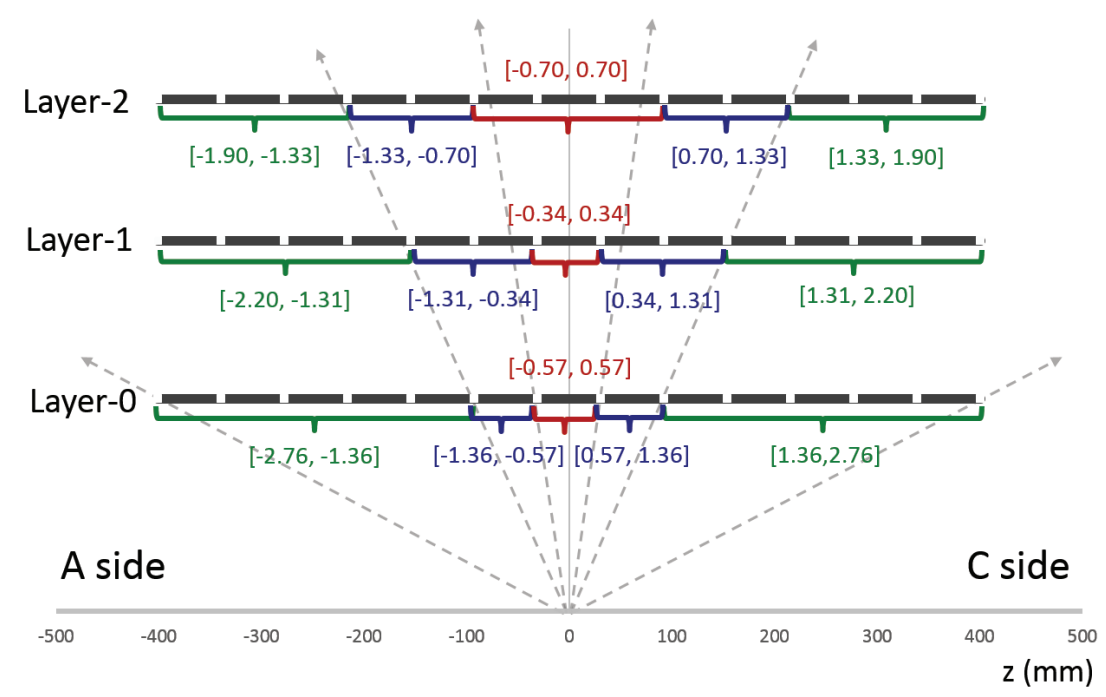

Figure 6: A diagram of the distribution of instrumented modules in each of five sectors of pseudorapidity.

integrated luminosity for Layer-0 in this scenario [10]. This extrapolation predicts a leakage current of about $500 \mathrm{~mA}$ per Layer-0 module (of thickness $250 \mathrm{~mm}$ ) after the accumulation of about 450 $\mathrm{fb}^{-1}$ of delivered luminosity, which corresponds to January 2024 in the scenario proposed here.

\section{Summary and prospects}

We have described the principles of radiation damage monitoring using the current measurements provided by the circuits of the ATLAS Pixel high voltage delivery system. We have observed radiation damage in the ATLAS Pixel Detector with characteristics in agreement with the Hamburg Model. The dependence of the leakage current upon the integrated luminosity has been presented. Linear behavior of the response has been observed. The current distribution in azimuthal quadrants is symmetric as expected. The shape of the current distribution in pseudorapidity is in agreement with a fluence calculation based on the FLUKA transport code within the assigned systematic uncertainties. Extrapolation of leakage current with time predicts survival of Layer- 0 up to $450 \mathrm{fb}^{-1}$ of data. This current monitoring system has been expanded to encompass pixel disk modules in Run 2 and beyond. 

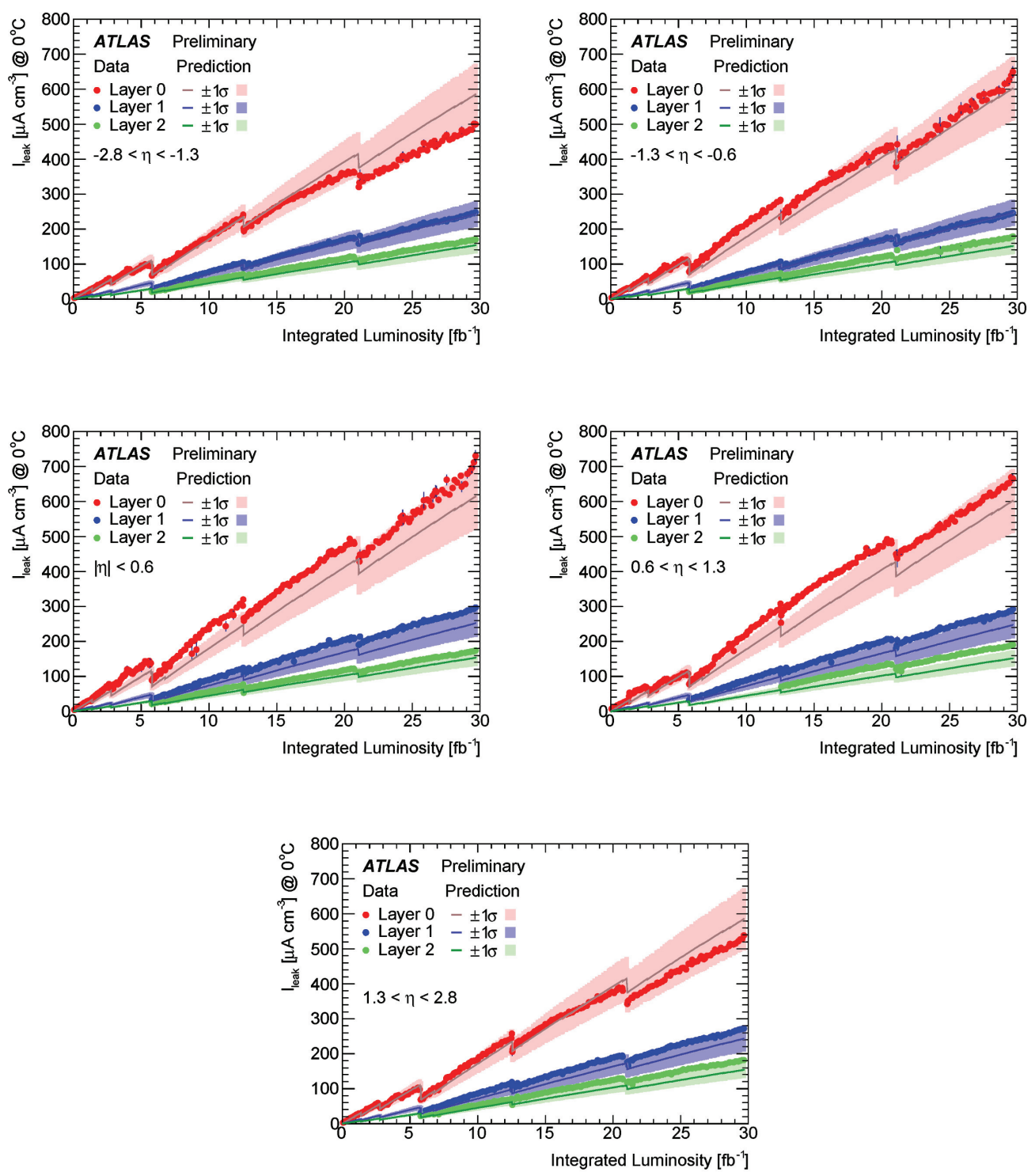

Figure 7: ATLAS pixel module leakage current in each of 5 pseudorapidity sectors, versus integrated LHC luminosity. The currents are averaged over their layer for all modules equipped with Current Measurement Boards within the layer. The current is continuously monitored by the ATLAS Detector Control System. Predictions based on the Hamburg Model are included. Discontinuities are due to annealing during LHC and cooling stops. 


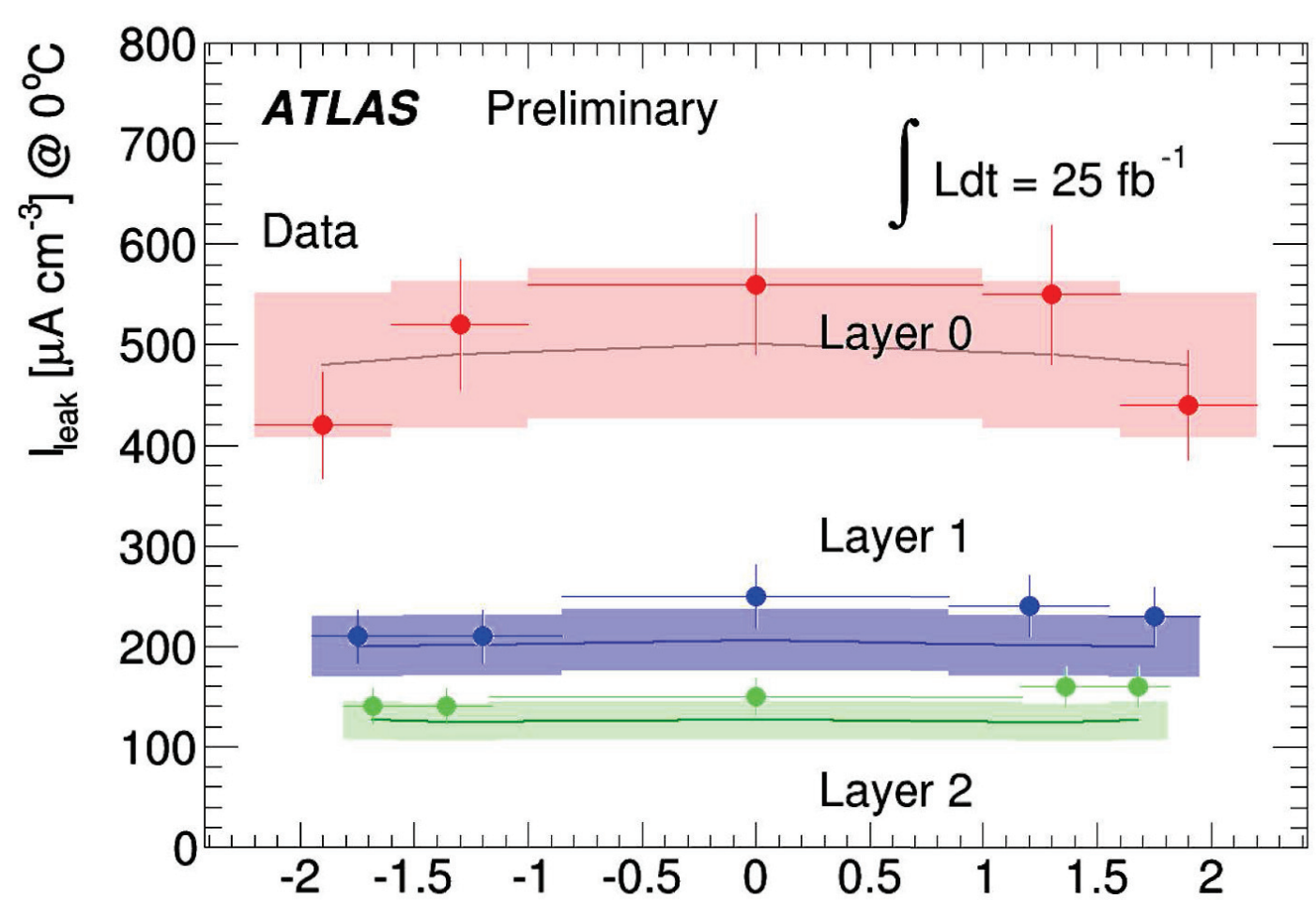

$\eta$

Figure 8: Module leakage current versus pseudorapidity, for integrated luminosity equal to $25 \mathrm{fb}^{-1}$. The currents are averaged over their layer for all modules equipped with Current Measurement Boards within the layer. The current is continuously monitored by the ATLAS Detector Control System. A prediction based on the Hamburg Model is included. 

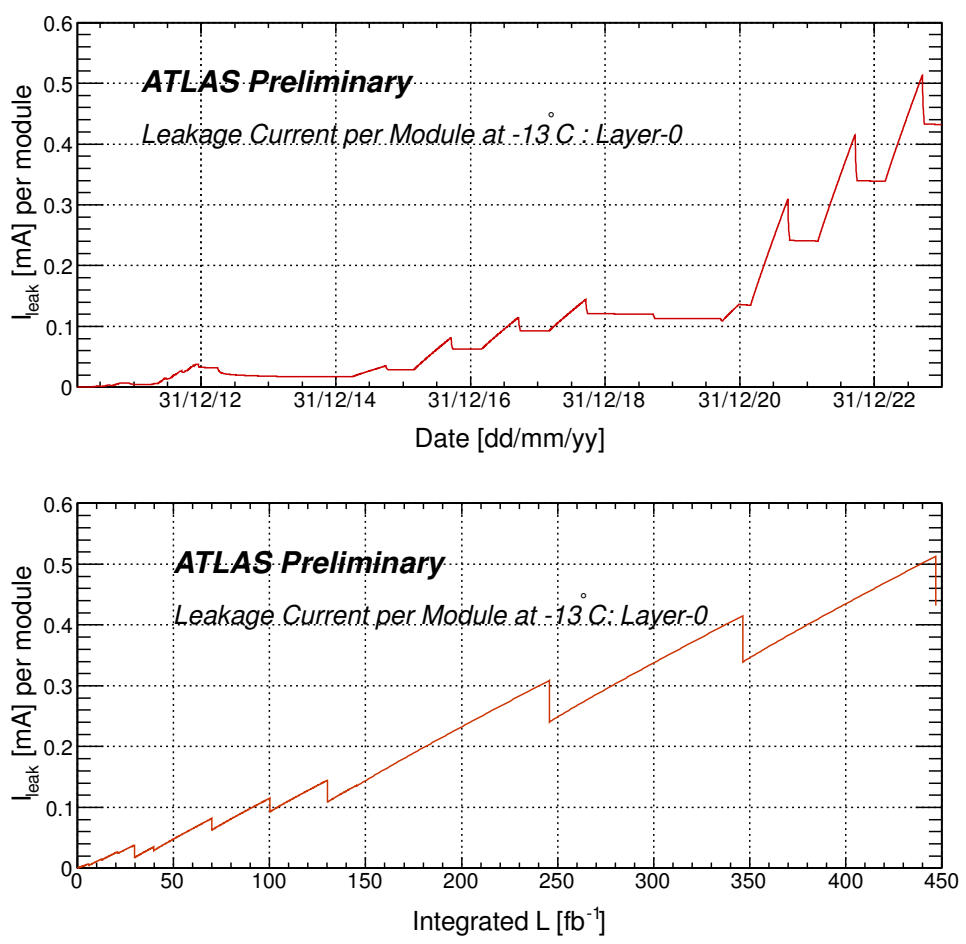

Figure 9: Predicted leakage current per Layer- 0 module at $-13^{\circ} \mathrm{C}$, versus date (upper) and integrated luminosity (lower) for the scenario in which modules will be exposed for 10 days to $20^{\circ} \mathrm{C}$ and otherwise maintained at $-13^{\circ} \mathrm{C}$. 


\section{References}

[1] G. Aad et al., ATLAS pixel detector electronics and sensors, 2008 JINST 3 P07007.

[2] ATLAS collaboration, The ATLAS Experiment at the CERN Large Hadron Collider, 2008 JINST 3 S08003.

[3] CERN-ROSE/RD48 collaboration, M. Moll, E. Fretwurst and G. Lindström, Leakage current of hadron irradiated silicon detectors - material dependence, Nucl. Instrum. Meth. A 426 (1999) 87.

[4] O. Krasel, Charge collection in irradiated silicon-detectors, Ph.D. Thesis, University of Dortmund, Dortmund Germany (2004), http://hdl.handle.net/2003/2354.

[5] S. Baranov, M. Bosman, I. Dawson, V. Hedberg, A. Nisati, and M. Shupe, Estimation of Radiation Background, Impact on Detectors, Activation and Shielding Optimization in ATLAS, ATL-GEN-2005-001 (2005).

[6] A. Ferrari, P. R. Sala, A. Fasso, and J. Ranft, FLUKA: a multi-particle transport code (program version 2005), CERN-2005-010, SLAC-R-773, INFN-TC-05-11, http://cdsweb.cern.ch/record/898301; G Battistoni et al., The FLUKA code: description and benchmarking, AIP Conf. Proc. 896 (2007) 31.

[7] T. Sjöstrand, P. Edén, C. Friberg, L. Lönnblad, G. Miu, S. Mrenna, and E. Norrbin, Computer Physics Commun. 135 (2001) 238.

[8] R. Engel, Photoproduction within the two component Dual Parton Model: amplitudes and cross-sections, Z. Phys. C 66 (1995) 203.

[9] A. Chilingarov, Temperature dependence of the current generated in Si bulk, 2013 JINST 8 P10003.

[10] ATLAS collaboration, A Leakage Current-based Measurement of the Radiation Damage in the ATLAS Pixel Detector, ATL-INDET-PUB-2014-004 (2014).

[11] Iseg Spezialelektronik GmbH, Rossendorf, Germany, http://www.iseg-hv.com.

[12] ATLAS collaboration, Improved luminosity determination in pp collisions at $\sqrt{s}=7 \mathrm{TeV}$ using the ATLAS detector at the LHC, Eur. Phys. J. C 73 (2013) 2518 [arXiv:1302.4393]. 